\title{
Inscrire la mémoire semi-nomade dans l'actualité sédentaire
}

Les églises de Pikogan et de Manawan

Marie-Pierre Bousquet et Anny Morissette

\section{OpenEdition}

\section{Journals}

Édition électronique

URL : https://journals.openedition.org/assr/12382

DOI : $10.4000 /$ assr. 12382

ISSN : $1777-5825$

Éditeur

Éditions de l'EHESS

Édition imprimée

Date de publication : 1 mars 2008

Pagination : 9-32

ISBN : 978-2-7132-2189-7

ISSN : 0335-5985

\section{Référence électronique}

Marie-Pierre Bousquet et Anny Morissette, «Inscrire la mémoire semi-nomade dans l'actualité

sédentaire », Archives de sciences sociales des religions [En ligne], 141 | janvier-mars 2008, mis en ligne le 02 juillet 2011, consulté le 08 juillet 2021. URL : http://journals.openedition.org/assr/12382 ; DOI : https://doi.org/10.4000/assr.12382 


\section{Marie-Pierre Bousquet, Anny Morissette \\ Inscrire la mémoire semi-nomade dans l'actualité sédentaire Les églises de Pikogan et de Manawan}

En Amérique du Nord, les églises ont figuré parmi les premiers édifices construits dans les réserves indiennes. Le Québec, qui fut une province canadienne ultra-catholique, n'a pas fait exception. Lors de la sédentarisation massive des Amérindiens encore semi-nomades de la province, entre les années 1940 et 1970, les réserves furent créées soit à partir de villages nés autour de postes de traite, où l'église figurait parmi les bâtiments, soit ex nibilo, sur des terrains où la structuration de l'espace en milieu villageois n'existait pas. Nous nous intéresserons ici à deux réserves relevant de la seconde configuration : Pikogan, réserve algonquine en Abitibi, et Manawan, réserve atikamekw en Lanaudière. Outre que les deux réserves se trouvaient dans la même province oblate ${ }^{1}$, elles représentent deux réalités géographiques différentes, qui ont de l'influence sur les modes de vie contemporains : Pikogan est proche d'une ville, Amos, alors que Manawan est isolée (à $72 \mathrm{~km}$ du village le plus proche). Dans ces deux communautés, l'édification des églises fut concomitante de l'érection des premières maisons. Rien d'étonnant à cela : au Québec, la prise en charge de la sédentarisation des Amérindiens a surtout été le fait des missionnaires oblats, ces derniers agissant comme médiateurs entre les chefs de bande et les instances gouvernementales (Gélinas, 2002 : 37 ; 2003a : 73 ; Leroux et al., 2004) et ce, depuis les années 1840 .

Pourquoi s'intéresser aux églises en particulier ? Depuis la fin des années 1960, le Québec a connu un mouvement de sécularisation lors de sa "Révolution tranquille ». Le taux de pratique chutant de façon vertigineuse, de nombreuses églises ont été abandonnées, ce qui a soulevé un débat public sur la place des biens d'Église dans la société. Si les églises sont les «châteaux » du Québec, monuments fondateurs de l'héritage culturel québécois, comme l'affirment Luc Noppen et Lucie Morisset (1996), qu'en est-il chez les Amérindiens ? Nous

1. Chez les Oblats, une province est une section de leur découpage administratif du territoire d'un pays. Ce découpage a été modifié dans les années 1980 : les provinces sont maintenant moins nombreuses, mais plus grandes. 
entendons montrer, à partir de l'exemple de Pikogan et de Manawan, que les églises des missions amérindiennes, lieux de mémoire polysémiques, sont des inscriptions physiques et symboliques d'un modèle de type post-colonial. De par leurs investissements contemporains, elles représentent aussi un début d'aprèscolonialisme.

Notre hypothèse de départ peut paraître curieuse. En effet, l'évangélisation des Amérindiens au Québec est couramment classée par les historiens et les anthropologues comme outil colonial. Par conséquent, les églises devraient en être le reflet bâti. Peut-être furent-elles, dans une certaine mesure, des modèles coloniaux, véhiculant et parachevant l'imposition d'une idéologie religieuse exogène, de la même façon que l'imposition du modèle du bungalow comme maison sédentaire fut un moyen de tenter de restructurer les habitudes de vie familiale semi-nomades (Casault, 2003). Mais le problème est plus complexe. En fait, il est délicat de vouloir séparer de façon tranchée ce qui serait de l'ordre d'un patrimoine colonial ou de l'ordre d'un patrimoine post-colonial. La limite d'un statut à un autre est relativement labile, tout comme l'est la limite entre le colonialisme et le post-colonialisme.

Le concept de "post-colonialisme » apparaît généralement dans les études sur les pays qui firent partie des anciens empires coloniaux (Appadurai, 2005 ; Memmi, 1957 ; Lazarus, 2006). Depuis l'ouvrage d'Edward Said (Orientalism, 1978), s'est ouverte une large réflexion sur l'impact de l'hégémonie ${ }^{2}$ coloniale sur les anciens colonisés, comme l'incorporation par les acteurs sociaux des stéréotypes produits sur eux pour les inférioriser et de l'image de civilisation supérieure véhiculée par le colonisateur pour renforcer son pouvoir. Appliqué aux Amérindiens du Canada, le post-colonialisme prend une nuance particulière : les auteurs laissent entendre que le Canada, qui a lui-même fait partie de l'empire britannique, est encore un État colonial. Ainsi, Poirier (2000) et Green (2004) montrent que la place des Amérindiens dans l'espace politique et économique canadien est conditionnée par leur nécessaire adaptation aux valeurs et aux rhétoriques de cet espace, déterminé par le pouvoir central. L'État, face aux revendications des Autochtones, aurait amorcé l'ouverture d'une voie post-coloniale, reconnaissant leur droit à l'autodétermination. Mais l'hégémonie politique est encore une réalité, s'exprimant dans les cadres juridiques et bureaucratiques dont les Amérindiens sont dépendants (Green, 2004 ; Ladner et Orsini, 2004 ; Long, 1990).

Nous retiendrons donc, d'entrée de jeu, de façon pragmatique, que le postcolonialisme est un stade où la domination est à la fois incorporée et profondément discutée et, en même temps, où les représentations et les pratiques coloniales sont réappropriées, de façon originale, par les acteurs concernés, alors que l'hégémonie politique se fait encore sentir dans les relations avec l'État. Les Amérindiens ontils achevé cette réappropriation ? Comment celle-ci se manifeste-t-elle dans leurs

2. L'hégémonie est ici comprise dans le sens que lui donnent les Comaroff (1991), à savoir une domination centralisée, incorporée et vue comme naturelle. 


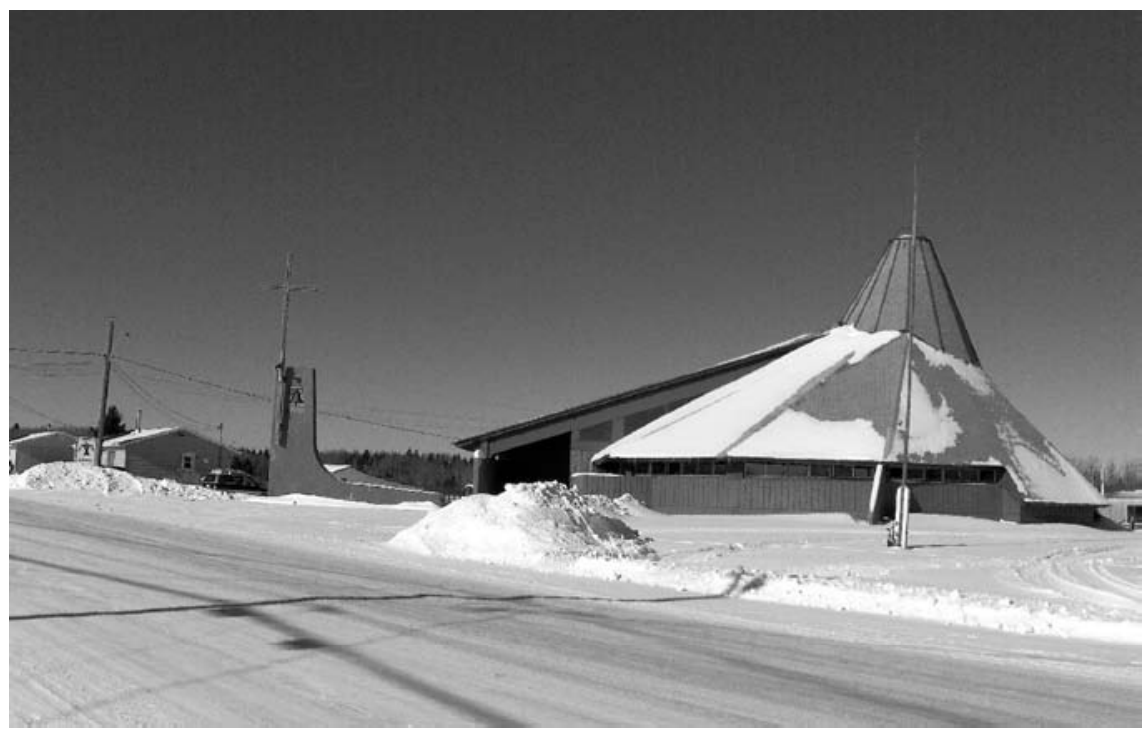

Église de Pikogan - Photo MPB-AM

usages contemporains des églises et de ce qu'elles contiennent ? Nous tenterons de montrer que ce que représentent les églises amérindiennes est à la fois de l'ordre du post-colonialisme et d'un début de décolonisation. Pour ce faire, nous avancerons que ces églises furent des théâtres de conversion à la sédentarité, puis qu'elles ont été, et qu'elles sont toujours, des expressions du patrimoine oblat autant qu'amérindien, enfin qu'elles sont aujourd'hui à la fois des lieux sacrés et désacralisés, porteurs d'une mémoire ambiguë. Pour appuyer nos dires, les données historiques ont été complétées par des observations et des entrevues de terrain ${ }^{3}$.

\section{La mémoire de la création des réserves}

L'inscription dans un milieu de vie sédentaire est un phénomène relativement récent pour les Amérindiens de Pikogan et de Manawan. Si, officiellement, la réserve de Manawan a été créée environ cinquante ans (1906) avant celle de Pikogan (1958), en pratique les familles commencèrent à y résider de façon permanente à partir de la fin des années 1950 pour la première, du début des années 1960 pour la seconde. Or, les Amérindiens étaient déjà convertis au christianisme

3. Marie-Pierre Bousquet effectue des recherches en milieu algonquin depuis 1996. Anny Morissette recueille des données chez les Atikamekw de Manawan depuis 2003. Pour la présente recherche, des séjours sur le terrain ont été complétés en janvier et février 2007. Nous tenons à remercier tous ceux qui nous ont prêté leur concours, en particulier André Mowatt, de Pikogan, et Gilles Ottawa, de Manawan, ainsi que le père Bertrand Bousquet et les Oblats œuvrant dans les missions ou travaillant aux Archives Deschâtelets. 
lors de cette installation. Ils avaient même intégré la religion catholique à leurs systèmes de représentations, au point de les considérer comme partie intégrante de leurs traditions (Bousquet, 2001; Gélinas, 2003b ${ }^{4}$ ). Ainsi, la construction de chacune des deux églises (en 1943 à Manawan, en 1968 à Pikogan) n'a pas été planifiée à des fins de conquête religieuse. Pour reprendre l'expression de Edgerton (2001), selon lequel la construction des missions et des églises dans le Mexique colonial a servi de " théâtres de conversion » dans l'entreprise de christianisation des Amérindiens du pays, les églises des missions amérindiennes du Québec ont plutôt été des théâtres de conversion à la sédentarité. En effet, non seulement elles ont été construites avec la participation active des membres des communautés, mais elles ont contribué à l'incorporation du modèle spatial du village tel que défini par les normes euro-canadiennes.

Les régions de l'Abitibi et de Lanaudière furent ouvertes à la colonisation forestière et agricole au courant du XIX $X^{e}$ siècle. Afin de contrer l'immigration des francophones catholiques vers les États-Unis et «maintenir au Québec une société rurale et traditionnelle où l'Église occuperait une place centrale " (Riopel, 1995 : 4), le clergé prit en charge cette colonisation, organisée autour de l'institution de la paroisse, "à la fois centre religieux et communautaire, lieu d'échange et de socialisation, [qui] permet l'identification et la cristallisation des valeurs collectives " (Linteau et al., 1989 : 207). Malgré les mutations économiques qui commencent dans les années 1950, les paroisses demeurèrent le fondement spatial des villages de ces régions, où l'église est située au cœur de la rue principale. De même, dans les réserves de Manawan et de Pikogan, quand les maisons commencèrent à sortir de terre, les missionnaires oblats, qui s'étaient installés en même temps que leurs ouailles, participèrent à l'organisation de l'espace. Le modèle du village euro-canadien fut reproduit, mais il ne faudrait pas en déduire que les oblats prirent l'initiative de faire bâtir les églises. Plutôt que d'avoir été les instigateurs des projets de construction et du choix des sites, ils n'en furent que les collaborateurs.

En effet, à Manawan, mission catholique depuis 1895 (Gélinas, 2003a : 26), le désir d'avoir une chapelle vint des Atikamekw, qui déposèrent leur requête auprès du père Guinard ${ }^{5}$ :

"Le Père Guinard leur répondit que c'était possible, à la condition que tout le monde travaille à la préparation des matériaux. Tout le monde a travaillé très fort pour bâtir cette chapelle durant quelques années. Lorsque la bâtisse fut prête en 1904, tous étaient très contents et fiers de leur travail " (ibid.)

Détruite par une tornade, en juin 1940, cette chapelle fut remplacée par l'église actuelle, terminée vers 1942-1943. L'inventaire des lieux de culte du

4. Peter Armitage (1992) et Denis Gagnon (2002) confirment aussi le fait pour les Innus.

5. Tiré du site La Nation Atikamekw de Manawan, http://www.manawan.org, consulté le 23 janvier 2007. 


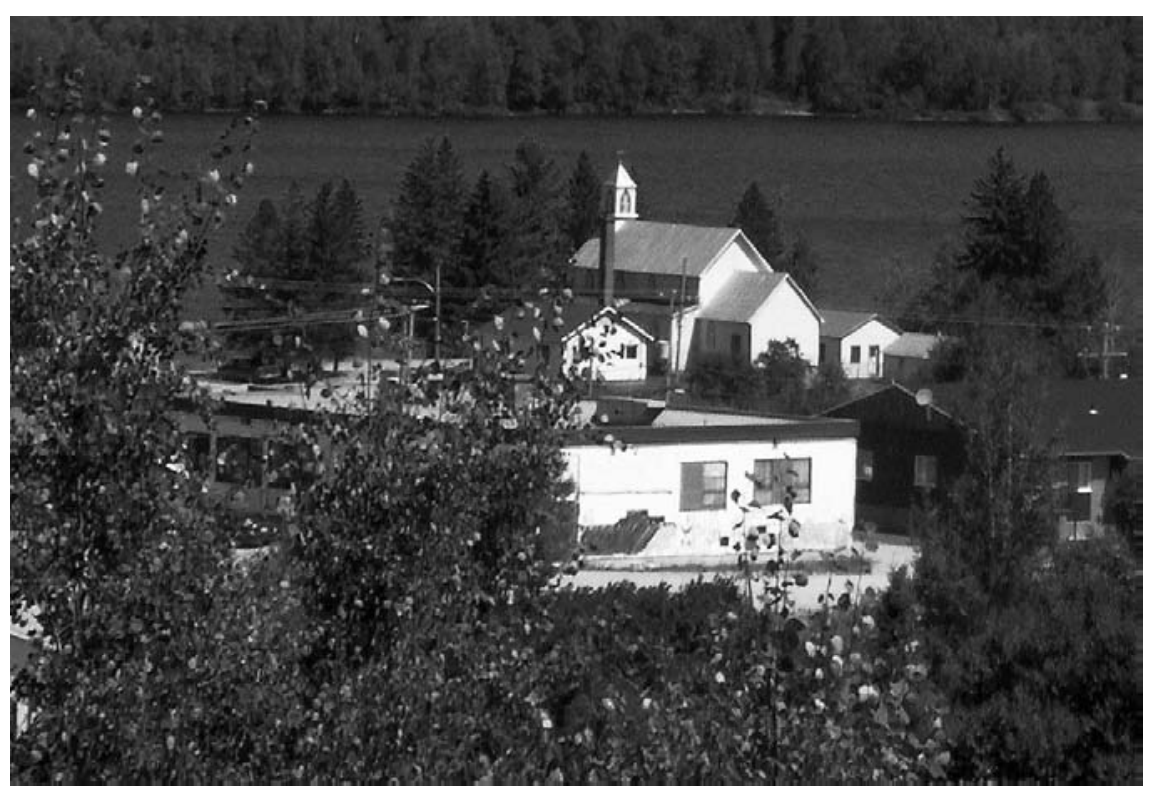

Vue de l'église de Manawan - Photo MPB-AM

Québec indique que le père Avila Sauvé fut le concepteur de cette église ${ }^{6}$. Là encore, les Atikamekw participèrent à la construction ${ }^{7}$. Elle possède une nef à trois vaisseaux, une tribune arrière ${ }^{8}$ et de hautes fenêtres provenant de la cathédrale de Trois-Rivières. Les premières maisons modernes de Manawan ayant été construites en $1951^{9}$, on peut dire que la chapelle, puis l'église, ont précédé l'apparition du village.

À Pikogan, les oblats fondèrent la mission catholique Sainte-Catherine en 1964, époque où les premières maisons de la rue principale furent construites. À la suite d'une concertation entre les Abitibiwinnik (nom des membres de la bande) et les oblats, il fut décidé de bâtir une église dont les fondations furent creusées pendant l'été 1967. Érigée d'après les plans de l'architecte Louis J. Lapierre (Anonyme, 1969 : 8), elle a la forme d'un tipi ${ }^{10}$. Elle fut inaugurée

6. Tiré du site Inventaire des lieux de culte du Québec, http://www.lieuxdeculte.qc.ca, consulté le 13 février 2007.

7. On compte notamment parmi les bâtisseurs Claude Flamand et César Moar, membres de la bande.

8. Tiré du site Inventaire des lieux de culte du Québec, op. cit. consulté le 13 février 2007.

9. Tiré du site Sur la route de Manawan, http://www.collections.ic.gc.ca/manawan.index. html, consulté le 23 janvier 2007.

10. Il s'agit, ici, non du tipi de type Indiens des Plaines, mais d'un habitat traditionnel algonquin, une petite tente conique recouverte d'écorce et de branchages. Cette tente est appelée pikogan, dont le nom est donné à la réserve en 1983 par une résolution du Conseil de bande. Précédemment, la réserve se nommait réserve indienne d'Amos. 
le 20 octobre 1968, "grâce à la collaboration de nos Frères et des Indiens qui ont su y mettre leur marque propre» (id.: 8$)$.

Il faut insister sur le fait que la participation active des Amérindiens du Québec à la construction de lieux de culte catholiques s'inscrit dans une continuité, celle du long processus que fut le passage à la sédentarité. En effet, Gélinas (2003b : 88-89), montrant la relation privilégiée qui s'était établie entre les missionnaires et leurs ouailles, rapporte « le zèle des Atikamekw à construire et à entretenir des chapelles à Kikendatch et à Weymontachie, leur contribution financière substantielle pour soutenir la mission, et le fait qu'ils n'hésitaient pas à braver les disettes dans l'attente de la robe noire ou dans l'espoir de demeurer plus longtemps en sa présence ». Jusqu'à ce que le semi-nomadisme touche à sa fin, les missionnaires ne rejoignaient les Amérindiens que l'été, sur les lieux de rassemblements estivaux traditionnels où s'étaient installés des postes de traite. En fait, la construction des chapelles dans ces lieux hautement signifiants pour la mémoire des bandes ${ }^{11}$ semble avoir été le fruit d'une stratégie des missionnaires : ceux-ci promettaient aux Amérindiens que, dès qu'elles seraient bâties, ils viendraient résider de façon permanente parmi eux (Carrière, 1958: 49 ssq.) Or, ce désir d'établissement permanent du missionnaire paraît avoir été réclamé par les Amérindiens euxmêmes qui, en divers endroits, offrirent dons et efforts physiques pour que ces lieux soient érigés. Au lac Abitibi, lieu de rencontre estival des Abitibiwinnik, la première chapelle fut construite en 1846 par des ouvriers qui n'étaient probablement pas amérindiens : " [le père Laverlochère] se rendit au Grand-Lac afin de laisser aux ouvriers le temps de bâtir la chapelle d'Abitibi. Il donna quatorze jours de retraite à Abitibi et durant les dix derniers jours la chapelle ne désemplit pas. Les Indiens ne cessèrent de chanter leur joie de savoir qu'ils avaient enfin une "sainte cabane de la prière" " (Carrière, $1963: 40)$. Si l'emphase de la rhétorique missionnaire est sans doute à relativiser, il n'en demeure pas moins que "en 1880, tous les membres du lac Abitibi collaborèrent à la construction d'une nouvelle chapelle» (Gosselin, $1996: 141$ ). Les incendies étant courants, une autre chapelle fut construite en 1911. Comme le site du lac Abitibi était peu propice à l'établissement d'une réserve, il fut peu à peu abandonné. Il ne subsiste rien aujourd'hui de ses bâtiments, mais la pratique d'avoir une chapelle à eux persista chez les Abitibiwinnik, fortement encouragés par les missionnaires qui préféraient les garder isolés des Blancs, dont la proximité était considérée comme nocive.

Établis en village, le rythme de vie des Atikamekw et des Algonquins fut désormais organisé selon le calendrier liturgique et non plus uniquement selon le rythme des saisons et des cycles migratoires des espèces parcourant leur territoire. Processions, célébrations en l'honneur des saints, la vie paroissiale était animée par le père oblat de leurs communautés respectives. L'église se mit à occuper une

11. Il est dûment attesté par les archéologues que les lieux de rassemblement estivaux des Amérindiens du Québec ont été utilisés de façon continue pendant plusieurs siècles. Chaque bande en tire d'ailleurs son ethnonyme. 
part croissante dans la vie des gens de Manawan et de Pikogan. Avant le départ à la chasse, une messe était célébrée où les hommes de Pikogan faisaient bénir leurs armes. Un aîné prenait la parole au cours de cette célébration, évoquant la chasse à venir, le territoire à exploiter ainsi que les prescriptions à suivre. L'église était un lieu de rassemblement non seulement dans le cadre de rituels chrétiens mais aussi dans le quotidien. À Pikogan, on venait même pique-niquer sur le terrain tout autour, à Pâques et pendant l'été. Ce terrain était perçu comme un espace pour tous, un lieu communautaire, et non comme un espace sacré où il aurait été sacrilège de pénétrer.

Plus que des lieux de culte, les églises de Manawan et de Pikogan sont des monuments à la sédentarité, à la vie permanente en communauté. Sont-elles des " géosymboles », selon la définition que donne Joël Bonnemaison (1996 : 167-168) de ce concept qu'il a créé ?

«Le géosymbole, expression de la culture et de la mémoire d'un peuple, peut se définir comme un lieu, un itinéraire, une construction, une étendue qui, pour des raisons religieuses, culturelles ou politiques, prend aux yeux des groupes ethniques une dimension symbolique qui les ancre dans une identité "héritée" ".

Le christianisme et la vie sédentaire sont le fruit de la colonisation des Atikamekw et des Algonquins. La construction de l'église, puis des réserves, témoigne de cette identité héritée. Ajoutant une nuance à la notion de Bonnemaison, Béatrice Collignon (2002: 60) considère les géosymboles comme étant des «lieux forts » de l'espace, comme il y a des « temps forts» de l'Histoire. Les églises de réserves sont à la fois des symboles de "lieux forts » de l'espace et de «temps forts » de l'histoire des Amérindiens. L'église de mission ne symbolise pas uniquement le commencement de la vie villageoise, mais aussi le premier lieu de mémoire non relié aux déplacements ou à un temps/saison de l'année. C'est un lieu fixe dans un temps continu. L'érection de l'église est concomitante de l'établissement en réserve des Atikamekw et des Algonquins, événement considéré par ces derniers comme étant le début d'une nouvelle période de leur histoire, un point zéro (Bousquet, 2005 : 9). L'utilisation constante de la formule " avant ", désignant la vie semi-nomade en forêt, et " après ", se référant à la sédentarisation et l'établissement en réserve, témoigne d'une rupture dans le temps social (Morissette, 2004 : 25). Puisque l'église de mission atteste de la relation des autochtones avec l'espace-temps, elle est aussi le symbole des liens unissant historiquement et physiquement les Amérindiens et les oblats de Marie-Immaculée, dans une charnière historique marquant l'ouverture d'une période post-coloniale.

\section{La création d'un art amérindien christianisé}

Les noms des deux missions témoignent d'emblée de l'histoire des relations entre les Amérindiens et l'Église. La mission Saint-Jean-de-Brébeuf de Manawan tire son nom d'un martyr canadien. Parmi les premiers missionnaires jésuites 
venus de France pour travailler à l'évangélisation de la Nouvelle-France, Jean de Brébeuf, fondateur de la mission huronne, est torturé et mis à mort par les Iroquois le 16 mars 1649 (Latourelle, 1999 : 22, 241). Canonisé par Pie XI en 1930, Jean de Brébeuf est proclamé patron du Canada par Pie XII en $1940^{12}$. Loin d'être ironique, la désignation d'une église de réserve d'après le nom d'un homme torturé par des Amérindiens est un témoignage de la dévotion à ses premiers missionnaires et de la continuité de leur œuvre au sein des missions amérindiennes et de leurs églises.

La mission Sainte-Catherine de Pikogan porte le nom de la première Amérindienne déclarée bienheureuse, Kateri Tekakwitha (Greer, 2005). Née vers 1656 d'une mère algonquine et d'un père mohawk, orpheline à six ans, elle est décédée à Kahnawake, près de Montréal, en 1680, quatre ans après avoir été baptisée par un père jésuite. Bien qu'ayant très tôt fait l'objet de récits hagiographiques, elle ne fut béatifiée qu'en 1980 par Jean-Paul II. Mais la dévotion envers elle, encouragée par les jésuites, puis par les oblats, fut marquée dès les débuts de la christianisation par la distribution aux Amérindiens d'images la représentant, images qu'on trouve encore fréquemment dans les intérieurs des maisons des réserves.

$\mathrm{Si}$, par ces deux noms, la toponymie catholique marque l'espace des réserves, c'est bien la mémoire de la vie semi-nomade qui marque l'intérieur de ces églises. Dès l'ouverture de l'église de Pikogan, avaient été installés dans son intérieur un chemin de croix en peaux de castor, un tabernacle en forme de tipi recouvert de peau d'orignal brodée de perles et une nappe d'autel, également en peau d'orignal brodée. CEuvres de sept femmes de la communauté, ces objets utilisent des matériaux et des motifs traditionnels, auxquels les artisanes ajoutèrent des lacets en cuir de vache ${ }^{13}$. Peu à peu, de nouveaux objets furent ajoutés, offrant le spectacle d'un mobilier et de vêtements ecclésiaux amérindianisés (croix en branches de bouleau, bénitier issu d'un tronc rongé par des castors et rapporté par un chasseur, paniers de quête en écorce, chasubles en peau d'orignal, étoles brodées de motifs animaliers, statue de la Vierge couronnée d'un bandeau à plumes, etc.). Des reliques de Kateri Tekakwitha furent aussi installées, dans des champignons durs qui poussent sur les troncs d'arbres (appelés ganodermes), dont les Algonquins se servaient pour transporter le feu.

De même, à Manawan, en 1972, suite à la réforme de Vatican II, l'autel fut déplacé pour que le prêtre puisse faire face aux fidèles. À cette occasion,

12. Dictionnaire biographique $d u$ Canada en ligne, http://www.biographi.ca/FR/ ShowBio.asp?BioId 34214, consulté le 15 février 2007.

13. Une ancienne guide touristique de Pikogan, petite-fille d'une des artisanes, expliquait que le cuir de vache avait été choisi "par respect envers les nouveaux arrivants " (faisant allusion aux colons fermiers). Il ne nous a pas été possible de confirmer l'hypothèse avec d'autres personnes, mais il est intéressant de noter, au moins sémiotiquement, l'effet du mélange entre la fourrure de castor et le cuir de vache, symboles de deux modes de vie, traditionnel et sédentaire. 


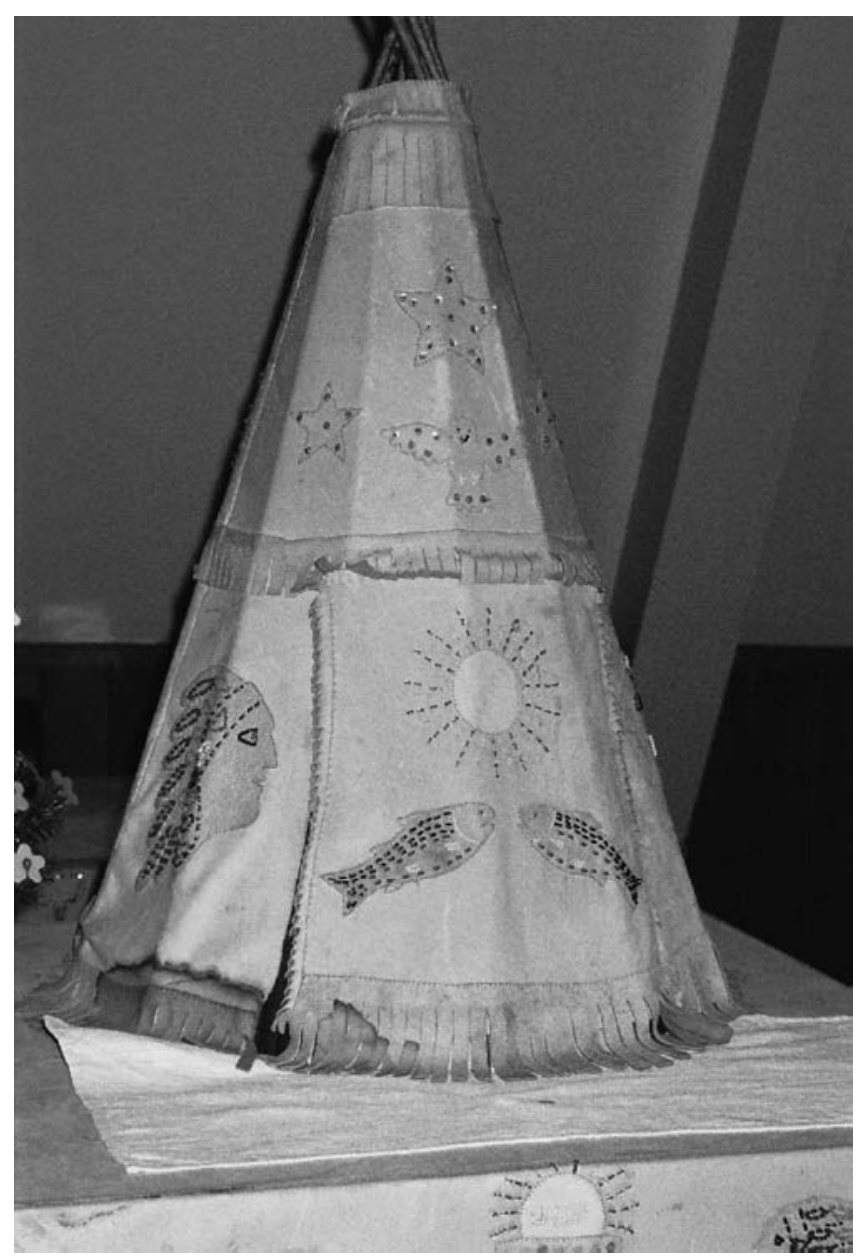

Église de Pikogan : tabernacle - Photo MPB-AM

les artisans de la communauté décorèrent l'église d'objets et de motifs types de l'artisanat et de la culture atikamekw. Un grand tipi en écorce fut placé derrière l'autel et un plus petit à son côté sert de tabernacle. Des motifs géométriques, animaliers et floraux ont été grattés sur la surface interne de l'écorce, exposée à l'extérieur des tipis, au même titre que des motifs religieux. Ces thèmes ornementaux se retrouvent sur une dentelle faite de bois qui orne le pourtour du chœur. Suspendue en son centre, une réplique miniaturisée d'un canot en écorce est datée de 1978. Les piliers de l'église ont aussi été pyrogravés d'animaux (notamment de poissons blancs ${ }^{14}$, de castors, de perdrix et d'orignaux), de fleurs,

14. L'ethnonyme Atikamekw signifie poisson blanc, corégone (McNulty, Gilbert, 1981 : 213). Il n'est donc pas étonnant de retrouver ce motif iconique représenté dans l'église de Manawan. 
d'arbres et de formes géométriques ${ }^{15}$. Le caractère amérindien est omniprésent dans chaque détail de la décoration. La forêt et la faune sont également présentes en ce lieu de culte (troncs d'arbres bruts utilisés comme pied de table ou de lutrin de lecture, excroissances d'arbres servant de support à statue, paniers d'écorce employés pour la quête et comme pot à fleur, nappes en peaux tannées et brodées de fleurs). Sur un mur de la mission St-Jean-de-Brébeuf, on trouve un portrait peint de Kateri Tekakwitha.

En introduisant au sein de la décoration de leurs églises des symboles de la vie semi-nomade, comme les animaux et les motifs floraux, les Algonquins et les Atikamekw insèrent une dynamique de mouvement sur le territoire dans ce lieu statique ${ }^{16}$. Béatrice Collignon (2002: 52-53) considère que le toponyme inuit est le vecteur de culturalisation d'un lieu, le récit du passé qui ancre la mémoire dans un espace "qui devient alors territoire ». Bien que les gens de Pikogan et de Manawan n'aient pas choisi le toponyme de leurs églises, ils se sont approprié ce lieu par l'intermédiaire de l'iconographie et de la création d'objets religieux originaux. L'art amérindien mis au service de la fabrication d'objets chrétiens marque spatialement l'église, qu'on reconnaît et fait sienne. On assiste de cette façon à la culturalisation d'un espace pour en faire un lieu de mémoire autochtone, un territoire symbolique.

Ainsi, ces églises font à la fois partie du patrimoine oblat et des patrimoines amérindiens. Symbolisant une continuité entre le passé semi-nomade et le présent sédentaire, leurs décors intérieurs et leurs mobiliers caractéristiques prolongent des arts de faire qui avaient émergé dès l'adaptation du catholicisme aux univers culturels des acteurs sociaux. En effet, en 1880, quand les Abitibiwinnik investirent leur nouvelle chapelle au lac Abitibi, « ils fournirent le matériel à sa décoration, dont une peau de castor, une peau de martre, etc. Le matériel utilisé pour la chapelle se comparait à [celui] que l'on utilisait pour le wigwam " (Gosselin, 1996 : 141). En contexte sédentaire, où le milieu n'était au départ pas signifiant pour les nouveaux résidents, ces objets et motifs conférèrent du sens à l'espace des réserves. Ils reflètent la particularité des lieux, qui se distinguent par ailleurs des autres villages du Québec moins par leurs formes que par les gens qui y vivent. Le témoignage de deux Huronnes, dans une brochure au sujet de l'église Notre-Dame-de-Lorette du Village-des-Hurons, paraît en confirmer l'idée (GrosLouis et Gros-Louis, $1983: 15)$ :

15. Alexis Quitish, membre de la bande de Manawan, est l'un des créateurs de ces pyrogravures.

16. Bien que les motifs ornementaux cités puissent être associés à un symbolisme magicoreligieux, les aînés interrogés sur leurs significations n'en ont pas fait mention, peut-être parce que cette partie du patrimoine a été oubliée. Ils ont juste mentionné que cela « faisait partie de leurs traditions". 


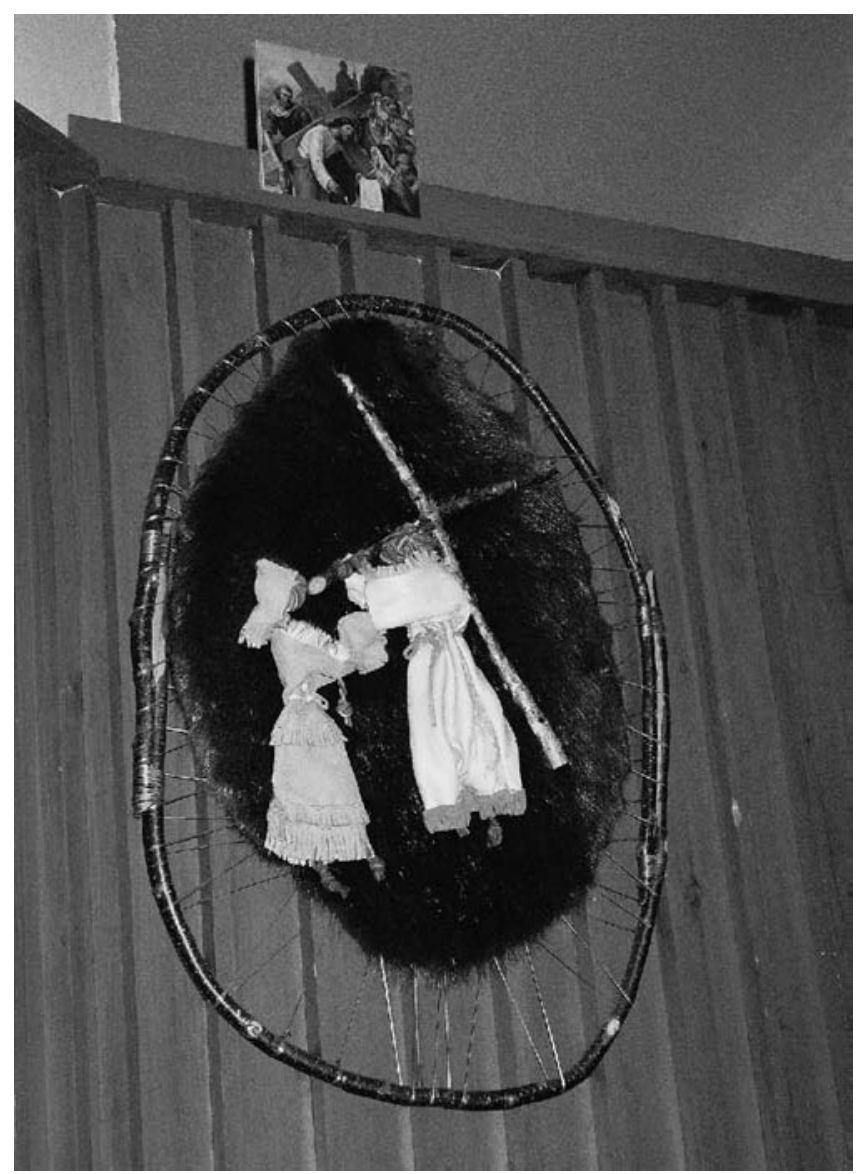

Église de Pikogan : chemin de croix : Ste Véronique Photo MPB-AM

«Les chandeliers de l'autel, le cierge pascal, les fonts baptismaux, l'ambon, le siège du célébrant et des servants de messe, le canot d'écorce renfermant les noms des familles; tout ce mobilier moderne indique que l'on se trouve sur une Réserve amérindienne dont les principales industries sont la fabrication de la raquette et du canot».

L'art amérindien christianisé, qui s'exprime à travers des matériaux vernaculaires, révèle les connaissances, les techniques, les habiletés propres à chaque communauté. Ces derniers aspects peuvent être considérés comme un "patrimoine religieux immatériel ", si l'on se réfère à la définition que donne Jean Simard (2004 : 172) de ce concept : «[...] l'ensemble des savoirs et savoir-faire qui ont permis ou permettent encore de donner vie et sens aux objets ». Mais il note aussi que "seuls les témoins vivants, porteurs de savoirs et de savoir-faire parce qu'ils en maîtrisent encore les codes, pourront détailler les fonctions et les noms de certains objets des liturgies chrétiennes [...] ». Or, dans le contexte actuel des 
réserves, non seulement la pratique catholique est devenue très faible, mais l'intérêt pour les objets religieux algonquins ou atikamekw n'est pas au premier plan des priorités de la sauvegarde culturelle. Puisque les générations qui ont participé à la création de cet art amérindien christianisé sont vieillissantes et que peu d'attention est prêtée à ces objets religieux, ces savoirs et savoir-faire sont en perdition dans les communautés autochtones.

La mémoire des membres des communautés n'est pas la seule à prendre en considération pour documenter l'art amérindien christianisé. Les pères oblats à la retraite, présents dans ces réserves au moment de l'érection des missions ou de leur rénovation, sont également porteurs de savoir. Plus largement, la mémoire de l'Église catholique permet de mieux comprendre les ressorts de l'émergence et du développement de l'art amérindien christianisé. En effet, c'est surtout à partir de la fin des années 1960 qu'on assiste à l'explosion de la fabrication d'objets catholiques de la part des Amérindiens dans les églises de missions et à l'émergence de nouveaux styles architecturaux (églises en forme de tipi ou de wigwam). Cette période correspond à l'après-Vatican II, concile qui a eu lieu en 1965. Or, Simard (2004 : 117-118) note qu'après Vatican II l'Église n'a plus besoin des artistes, sculpteurs, orfèvres, peintres, brodeuses, ferronniers, puisqu'elle « n'est que la maison du peuple » : "Il reste néanmoins que l'Église catholique du Québec n’a plus besoin des artistes pour la raison bien simple qu'elle ne désire plus présenter ses temples comme des miroirs du ciel ». Nous pensons que cette assertion doit être nuancée. Vatican II, prenant en compte les aspirations à mettre en valeur la diversité des cultures, a encouragé le développement de nouveaux arts, pour des raisons de recherche d'ouverture. L'Église a donc eu besoin de nouveaux artistes. S'inscrivant dans ce courant, les Oblats ont favorisé l'utilisation de symboles des traditions amérindiennes pour traduire des réalités chrétiennes. Cette préoccupation n'était pas nouvelle pour eux, mais elle s'est fait sentir avec plus d'acuité après Vatican II : "L'ornementation de nos églises a-t-elle quelques reflets qui plaisent aux Indiens ? ", écrit ainsi le père Rémi Côté, o.m.i., en 1967.

Même si l'Église semble avoir joué un rôle, il n'en demeure pas moins que les artistes amérindiens ont choisi eux-mêmes ce qu'ils voulaient représenter. Ils ont créé pour leurs églises une série d'objets qui, par leur originalité et leur évocation du mode de vie semi-nomade, constituent une importante contribution au patrimoine religieux global du Québec. Les églises de missions et l'art amérindien christianisé sont devenus des trésors nationaux, reflétant un patrimoine complexe, à la fois catholique, euro-canadien et amérindien. En effet, comme l'explique Achiel Peelman (2004 : 132) :

«[...] nous devons reconnaître que l'Église amérindienne est loin d'être une Église vraiment inculturée. Certes, elle utilise les langues autochtones dans sa liturgie et dans sa catéchèse. Elle emprunte aux cultures autochtones toutes sortes d'éléments matériels (arts, symboles, chants, musiques) pour créer un environnement communautaire qui permet à ses membres autochtones de se sentir chez eux. Par contre, dans ses aspects 
fondamentaux, elle demeure une copie de l'Évangile catholique, "universelle et romaine”, qui est venue s'implanter parmi les peuples premiers de l'Amérique du Nord ".

Peelman semblerait donc nier que les églises amérindiennes soient passées d'un stade colonial à un stade post-colonial, si l'on entend par là que le point de vue qui domine reste, envers et contre tout, celui de l'Église catholique. Sans doute ce théologien a-t-il raison sur le plan de la liturgie. Mais nous pensons que ce n'est pas le cas d'un point de vue patrimonial. Si l'on peut dire, les Amérindiens ont "acculturé " leurs églises au sens où ils ne les utilisent plus, ou du moins pas seulement, à des fins religieuses et sacrées.

\section{Des lieux sacrés et désacralisés}

Bien que restant d'abord et avant tout des lieux sacrés, de par leur fonction première, les églises des missions amérindiennes sont, en ce début de XXI ${ }^{\mathrm{e}}$ siècle, désacralisées. Comme il a été dit plus haut, la pratique du catholicisme a considérablement diminué, au point que l'église de Pikogan, n'ayant plus assez de paroissiens assidus pour justifier la présence permanente d'un missionnaire, a été transférée par les oblats au conseil de bande de la communauté (celle de Manawan a été prise en charge par le diocèse de Joliette). Il semble en fait que la notion de lieu de mémoire de Pierre Nora puisse s'appliquer aux églises dont il est question ici. Comme le rappelle Willaime (1988 : 126), Nora, en définissant le lieu de mémoire, replaçait l'entreprise "dans le cadre d'une problématique de la désacralisation ": la nation, ne croyant plus à son " histoire sainte ", congédie son passé et patrimonialise des lieux qu'elle a désacralisés, afin de compenser la perte de sa "mémoire traditionnelle ». S'y adjoint une dimension d'ordre politique, d'interprétation de l'expérience historique à des fins de légitimation d'un ordre qui la transcende et l'institutionnalise.

Investies d'une double mémoire, celle des oblats et celle des Amérindiens, ces églises sont devenues des lieux traversés de dimensions contradictoires. Parler des oblats, dans une communauté algonquine ou atikamekw, c'est d'abord parler d'individus plus que d'une congrégation : parmi ces hommes, certains ont laissé d'excellents souvenirs, d'autres de terribles. C'est aussi parler de l'abandon du chamanisme, que les aînés ont connu mais dont ils n'aiment guère parler, ce que les jeunes regrettent. C'est encore parler d'une conversion des ancêtres que leurs descendants tentent de comprendre et dont beaucoup pensent, a posteriori, qu'il s'agissait d'un « lavage de cerveau » : «Là-bas [Pikogan], les gens parlent beaucoup mais ne font rien à cause du brainwash catholique. Il n'y a plus d'énergie » (Montpetit, 1993 : 128). Sont alors évoquées les humiliations, les sanctions et les punitions qui ont marqué la mémoire orale ${ }^{17}$ et que les écrits du père Proulx

17. Une dizaine d'aînés interrogés entre 1997 et 2007 se souviennent même des noms des missionnaires réputés pour leur sévérité et de ceux réputés être plus gentils. 
(1882 : 242) confirment quand il est question des missions de Wemontaching (Wemotaci). Ces techniques ont également été employées par le père Guéguen pour convertir les Algonquins du Grand Lac (Carrière, $1978: 120)^{18}$. C'est enfin parler des séquelles douloureuses des pensionnats indiens, qui faisaient partie du programme fédéral d'assimilation des autochtones (Dion Stout, Kipling, 2003). Au Québec, ces écoles résidentielles fonctionnèrent entre le milieu des années 1950 et le milieu des années 1980 et étaient confiées aux oblats ${ }^{19}$ (Bousquet, 2006). Depuis leurs fermetures, ont émergé des demandes de réparations pour les préjudices subis par les anciens pensionnaires, mettant en évidence les affaires de pédophilie ${ }^{20}$.

Mais parler des oblats et de l'Église dans une réserve, c'est aussi parler du mode de vie semi-nomade qu'ont connu les gens les plus âgés, d'un temps où l'ordre social régnait, selon les règles, alors acceptées, de la foi catholique. Comme l'indique l'autobiographie d'un aîné algonquin, à partir du moment (1844) où les oblats investirent chaque été les lieux de rassemblements estivaux, les événements les plus importants de l'année devinrent les fêtes chrétiennes :

"L'Église [...] était omniprésente dans notre vie. [...] La Big Mission était le point de ralliement de tous les Anishinabeg ${ }^{21}$. [...] Des centaines et des centaines de personnes se retrouvaient sur place. La célébration en l'honneur d'un Saint ou d'une Sainte, comme Sainte-Anne, était véritablement un grand jour. La fête de Sainte-Anne était en fait l'événement le plus important de l'année. La messe était suivie d'une procession qui partait de l'église pour remonter tout le village jusqu'au lac. Les arbres étaient ornés de drapeaux et de bannières, et nous chantions divers hymnes et chants religieux et priions tout au long de la marche. Le prêtre, qui marchait avec nous, se servait de l'encens et de l'eau bénite pour bénir tous les gens et toutes les créatures vivantes. Il me semble qu'en ce temps-là, le sentiment religieux était beaucoup plus fort qu'aujourd'hui » (Mathias, $1998: 40$ ).

Quand les aînés évoquent ce temps-là, où les enfants étaient baptisés dès leur naissance, où les jeunes gens se mariaient avec le consentement de leurs parents et où les préceptes réglant le bon fonctionnement de la vie sociale étaient respectés, la comparaison avec la situation contemporaine est au détriment de celle-ci.

18. Gagnon (2002 : 54) atteste également le fait chez les Innus puisqu'il relate, pour la mission innue de Musquaro, des pratiques de "mise à l'écart, prosternation et longues heures passées à genoux ", ainsi que "la menace de l'enfer et du démon [...] régulièrement utilisée comme mesure disciplinaire ", des années 1840 à 1940.

19. Il y eut six pensionnats indiens au Québec. En fait, deux ouvrirent avant la Seconde Guerre mondiale et l'un d'entre eux était géré par les anglicans.

20. Il faut être conscient que les opinions des Atikamekw et des Algonquins au sujet des pensionnats qu'ils ont fréquentés ont évolué au fil du temps : de tabou, le sujet a été fortement médiatisé après le rapport de la Commission royale des peuples autochtones en 1996 et la création, en 1998, de la Fondation autochtone de guérison. Cette médiatisation a marqué les consciences au point qu'idéologiquement, les séquelles douloureuses ont tendance à prendre le dessus sur l'ensemble des souvenirs.

21. Anishinabe, ou Anicinabe (pluriel -eg ou -ek) est le nom vernaculaire des Algonquins. 


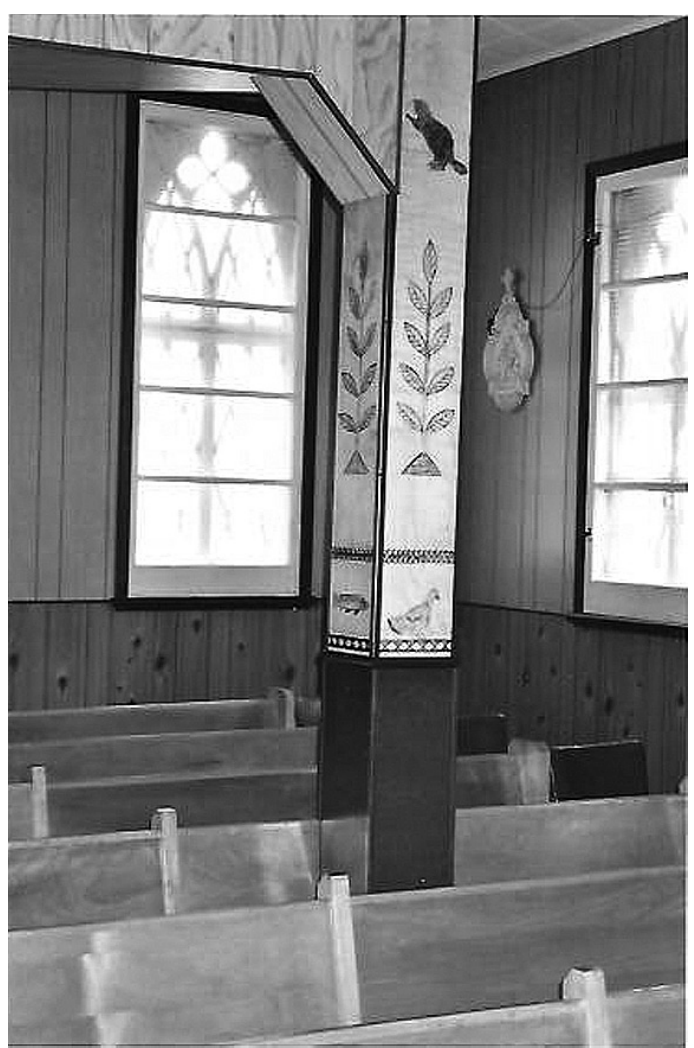

Église de Manawan : pilier - Photo MPB-AM

Certes, les aînés peuvent être virulents contre les prêtres, mais conçoivent le catholicisme comme faisant partie de leur culture. Tout n'est pas relatif à leur foi religieuse : ils ont été élevés par leurs parents dans le respect des paroles des missionnaires, dans l'idée que les croyances de leurs ancêtres n'étaient que superstition et sorcellerie. Outre qu'ils ne renient pas l'éducation qu'ils ont reçue, ils se souviennent que la remise en question de l'Église a plusieurs fois déclenché dans les communautés des vagues de violence et de division. L'ambiguïté de la place de l'Église comme institution et des églises comme lieux ressort de leurs discours, ainsi qu'en témoignent ceux prononcés lors d'une réunion d'aînés de la bande du Lac-Simon, en 1996 :

«Avant, les gens trouvaient leur force à l'église. C'était un lieu de retrouvailles. Mais les églises sont désertées. Il n'y a plus de place pour se parler. Heureusement, maintenant, il y a des cercles de parole et de guérison. Le cercle donne de la force car on peut y communiquer. Sinon, les Anicinabek n'auraient plus rien pour se parler» (Bousquet, $2001: 382$ ).

Quand les aînés parlent de l'écart entre le passé, monde de l'équilibre, et le présent, monde du désordre social, ils font constamment référence à l'espace, 
divisé en deux parties : les lieux de communication (la forêt, lieu idéal de vie et d'expression des valeurs, et l'église) et les lieux de non-communication (la réserve, la ville). Mais, dans leur classification de ce qui est et qui n'est pas un lieu algonquin, la répartition peut être ambiguë :

«Seules les églises, en tant que lieux, n'arrivaient pas à être rangées dans les catégories algonquin-non algonquin. [...] Pour eux, l'église est plutôt un lieu entre deux, mi-blanc mi-algonquin » (Bousquet, $2001: 383$ ).

Ainsi, ces églises sont conjointement des symboles d'un passé d'adhésion collective au catholicisme qui doit être assumé et d'un présent qui passe plus ou moins, oscillant entre la revendication d'un ancien ordre social revitalisé et la négation d'une partie de cet ordre. Les oblats, de leur côté, prennent acte de cette ambiguité : s'ils reconnaissent que les membres de leur congrégation ont commis des erreurs, ils trouvent le jugement des Amérindiens sévère à leur égard : " N'avons-nous fait que du mal ? " Mais ils constatent aussi que, contrairement à ce qui s'est passé dans d'autres pays, il n'y a eu que de très rares vocations à la prêtrise chez les Amérindiens.

Les églises des missions amérindiennes, désertées par leurs paroissiens, abandonnées par les missionnaires, ne sont-elles donc plus des lieux cultuels ? Il serait prématuré de l'affirmer. Depuis quelques années, le mouvement catholique du renouveau charismatique est présent à Manawan, de même que dans d'autres communautés amérindiennes (Laliberté, 1998 ; Grenier, 1998). Ce mouvement consiste en des soirées de prières animées par des gens de la communauté où les participants prennent la parole, ainsi que des retraites de fin de semaine à l'extérieur de la communauté. Ces soirées et retraites font partie d'une démarche de guérison pour les autochtones (Laliberté, 1998). D'après le père Laliberté, o.m.i., le renouveau charismatique a amené un "réveil spirituel » dans les communautés atikamekw, où la pratique religieuse semblerait connaître un regain. Pourtant, dans un article du journal L'Action, le chef de la bande de Manawan, Paul-Émile Ottawa, remarque que «Comme partout au Québec, la foi n'est plus ce qu'elle était. Il y aura par contre du monde à la messe de Noël [...] " (Pelletier, 2006).

En fait, les églises de missions ont tendance à s'autochtoniser : si la foi «n'est plus ce qu'elle était » et les missionnaires ne résident plus que rarement dans les communautés, la volonté de perpétuer le catholicisme n’a pas disparu. À Manawan, comme « le tiers du temps, le prêtre n'est pas présent dans la communauté ", un membre de la bande préside les célébrations du dimanche (Laliberté, 2001 : 11), phénomène qui existe dans d'autres missions oblates chez les Amérindiens (Cadieux, 2003 : 10). Ainsi, par l'engagement pastoral et liturgique, on peut dire que, d'un point de vue spirituel et religieux, les communautés sont entrées dans une voie post-coloniale.

Ce processus est encouragé par les oblats, dont la revue Apostolat se fait l'écho. Les oblats ont tenu des tables de concertation avec les Amérindiens, où ils affirment « [...] non seulement qu'il y a une Église chez les Autochtones, mais 
INSCRIRE LA MÉMOIRE SEMI-NOMADE DANS L'ACTUALITÉ SÉDENTAIRE - 25

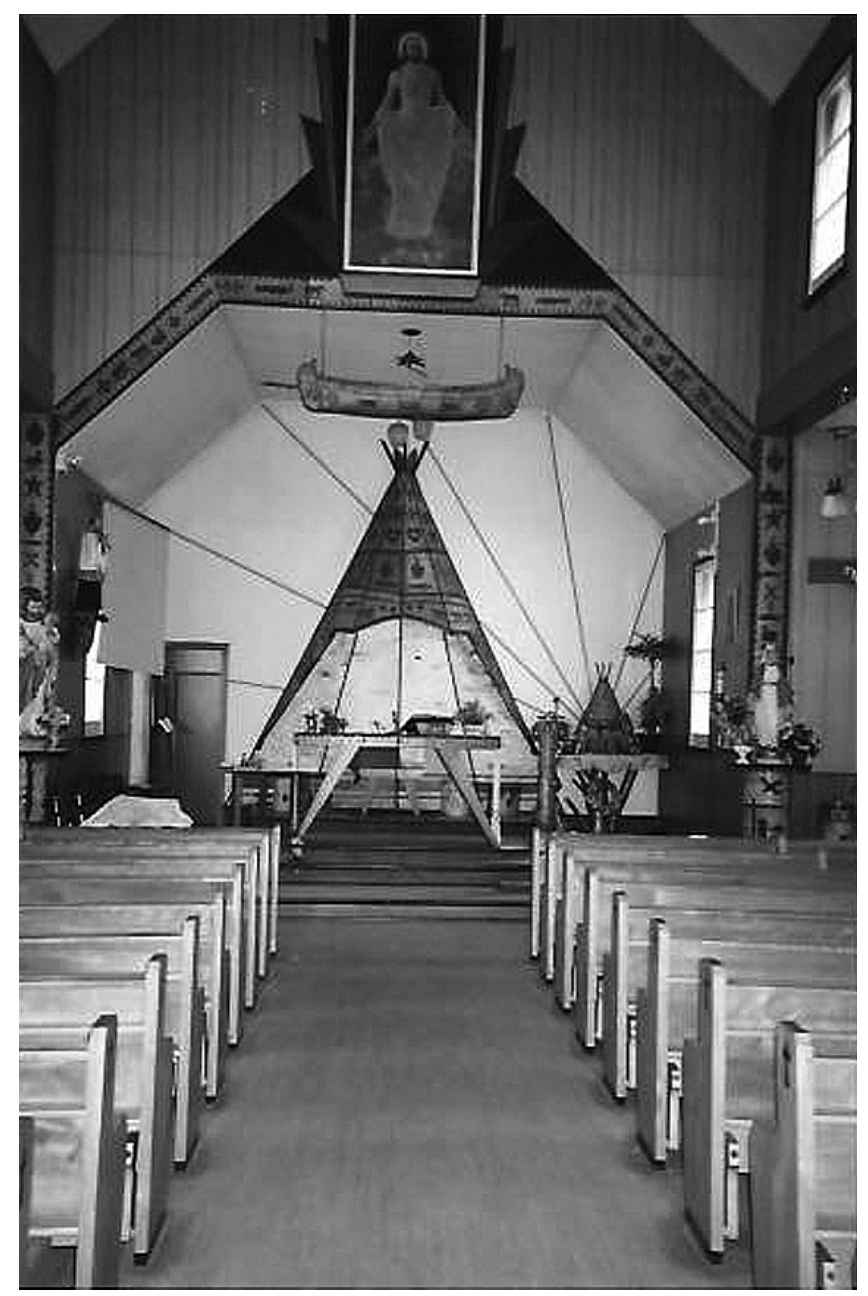

Église de Manawan : l'autel - Photo MPB-AM

aussi qu'il y a une Église autochtone »(Laliberté, 2005 : 6). La délégation des rôles des missionnaires aux Amérindiens y est vue de façon positive, pour participer à un mouvement émancipatoire qu'on pourrait qualifier de "décolonisateur " : " Notre expérience auprès des autochtones nous dit qu'aussi longtemps qu'il y aura quelqu'un de l'extérieur pour remplir certaines tâches, ces derniers n'oseront pas s'engager (Cadieux, 2003 : 10) ». S'il y a une prise en main de l'église par les autochtones au même titre qu'une autochtonisation de l'Église, c'est aussi parce que les autochtones " [...] croient et pratiquent à leur façon » (Lapointe, 2005 : 10). Mais cette " délégation " n'est pas toujours vue comme une émancipation par les membres du clergé : en fait, les missionnaires aujourd'hui n'ont pas vraiment le choix. Ils sont souvent très âgés et ne trouvent pas de remplaçants. 
Ainsi, ils prennent acte de leur mise à l'écart, venant jouer des rôles de célébrants de cérémonies quand ils y sont invités, mais peu consultés par leurs anciennes ouailles sur l'avenir des missions dont ils ont été responsables.

Mais si les églises de missions continuent d'être des lieux de rassemblement de ce qui reste de croyants, elles sont surtout devenues des lieux culturels. Comme les Amérindiens ne peuvent modeler les réserves à leur guise ${ }^{22}$, les églises sont d'importants lieux d'expression de leur mémoire d'un temps ancien et actuel. Ainsi, elles constituent un des premiers endroits où l'on emmène le visiteur de passage. À Pikogan, où le tourisme fait partie des priorités de développement économique, la Société Amik organise des circuits d'excursion dont l'église Sainte-Catherine est un des piliers. L'église, son tabernacle et son chemin de croix figurent sur la première page du dépliant de la Société et sur des cartes postales. Elle est aussi mentionnée sur tous les livrets et sites internet de promotion de la région de l'Abitibi-Témiscamingue, avec partout le même court texte d'accompagnement :

«L'église de Pikogan est aujourd'hui plus qu'un lieu de culte, c'est une rencontre avec l'histoire et les réalités de la nation qui a joué un rôle important dans l'histoire régionale et qui contribue aujourd'hui au développement de l'Abitibi-Témiscamingue ».

Ainsi, l'église est réappropriée par les membres de la bande de Pikogan, par le biais des visites touristiques qui sont sous leur contrôle et par le biais des discours des guides locaux, qui décident de ce qu'ils veulent montrer et interpréter. L'accent est alors mis sur le travail des artistes et sur la métaphorisation de la vie semi-nomade plutôt que sur l'Église catholique en elle-même. Dans ce contexte, les objets et les motifs décoratifs ont des fonctions commémoratives : ils servent de support à une interprétation politisée de l'histoire de la bande, légitimant le nouvel ordre où la communauté contrôle son devenir religieux. À Manawan, communauté plus isolée où le tourisme est plus factuel qu'organisé, l'église est, mais dans des proportions moindres, pareillement investie. Dans des villages où le patrimoine bâti, principalement constitué de bungalows et de bâtiments communautaires (postes de police, conseils de bande, écoles), est jugé localement comme pauvre, les églises et ce qu'elles renferment sont des atouts potentiels de mise en valeur de soi et de sa spécificité culturelle, sociale et historique.

Le sens de cet investissement, s'il leur échappe parfois, est noté par les oblats. Ainsi, le père Desaulniers, o.m.i. (2005 : 16), qui œuvre chez les Innus de Maliotenam, remarque :

22. Rappelons que les schèmes d'établissement des réserves, ainsi que l'érection de maisons, ont été conçus par des non-Amérindiens. Les réserves amérindiennes sont sous la fiducie du gouvernement fédéral. Comme, dans la plupart des cas, les maisons des réserves appartiennent au conseil de bande et, par le fait même, au gouvernement fédéral, les Amérindiens ne peuvent apporter aucune modification à ces propriétés, pas même l'ajout de couleurs sur les murs. 
"Que de fois j'ai vu des gens, jeunes et âgés, hommes et femmes, assis immobiles, ils regardaient, pendant de longs moments, peinte sur les murs de cette église, une fresque de la vie et de l'environnement autochtones. Ils étaient en pleine contemplation. Que se passe-t-il dans leur tête ? Quelles histoires reviennent à leur mémoire ?"

À l'heure actuelle, la signification des églises sur les territoires des réserves amérindiennes est au cœur d'un processus de mutation, où les communautés se cherchent à nouveau des valeurs communes à partager et se trouvent en situation de redéfinir leurs traditions. Les Algonquins et les Atikamekw ne sont plus des semi-nomades. Sont-ils encore catholiques ? S'ils ne le sont plus, que sont-ils ? Les églises de missions symbolisent l'ensemble de ces dilemmes où se discute la position de "ni vainqueurs, ni vaincus " (Laugrand, 1997) des Amérindiens face aux missionnaires qui ont fait partie de leurs colonisateurs.

\section{Conclusion}

La vie en communauté et la sédentarité sont des phénomènes relativement nouveaux pour les Algonquins et pour les Atikamekw. Du temps du semi-nomadisme, les familles qui formaient une bande ne se retrouvaient que durant la saison estivale. Vivre dans des villages, qui plus est sous contrôle fédéral, a impliqué de nouvelles règles de gestion sociale et les Amérindiens ont dû apprendre à penser avec le système. Aujourd'hui, ils travaillent, peu à peu, à se réapproprier des pouvoirs décisionnaires. Si les églises de missions ont pu contribuer à implanter un sentiment communautaire, fédérant les paroissiens autour de ses activités, elles ont progressivement été évincées de l'espace politique (entendu ici comme espace de discussion des valeurs et des normes). Le pouvoir hégémonique des prêtres, qui fut autant spirituel que politique ${ }^{23}$, n'existe plus. Les Amérindiens reprennent leur destinée en main et, dans ce processus, ils ont aussi écarté les missionnaires. Ceci se traduit autant dans la conduite des rituels que dans l'investissement des églises : il arrive, de nos jours, que les prêtres trouvent, en venant ouvrir le bâtiment, des objets, comme des statues, rapportés ou créés par des résidents de la réserve. L'implantation de type post-colonial d'un modèle de civilisation catholique euro-canadienne est en voie de se transformer en symbole d'après-colonialisme, voire, mais cela restera à prouver, de décolonisation.

Dans des contextes de réserves amérindiennes, où la superficie est figée mais où la population ne cesse de croître, on peut craindre pour l'avenir de certaines églises de missions. L'espace aménageable étant restreint, soit l'église est recyclée en centre culturel ou en lieu d'attraction touristique, soit le bâtiment est menacé de destruction pour laisser place à d'autres projets. La protection du patrimoine bâti est une préoccupation importante au Québec. Dans ce patrimoine, les bâtiments catholiques, désertés, font l'objet d'une attention particulière depuis les

23. Les missionnaires se sont parfois arrogé, de facto, le rôle de chefs (Gélinas, 2002). 
années 1980, ce qui, selon Tania Martin (2007 : 42), reflète la place du catholicisme dans le paysage. Mais ces questions sont, à l'heure actuelle, loin de faire partie des priorités des communautés amérindiennes.

Dimanche matin, Pikogan, le 24 septembre 2006. Il règne ce jour-là une animation inhabituelle à l'église depuis le début des années 2000. Au lieu de touristes, des gens de la communauté affluent. L'émission « Le Jour du Seigneur ", de Radio-Canada, s'est arrêtée, pour quelques heures, dans la réserve. À l'annonce de l'arrivée des caméras, les quelques fervents catholiques locaux ont tenté de rameuter des membres de leur parenté en grand nombre, mais en vain : les caméras seront obligées de tourner pour donner l'impression que l'église est remplie. Mais l'émission donnera l'occasion aux responsables du centre culturel de s'exprimer sur les relations avec les oblats et avec le catholicisme, ainsi que sur la sauvegarde de la culture. Ainsi, la domination des prêtres est chose du passé et, dans le même temps, les représentations et les pratiques associées au christianisme sont rejetées, ce qui amènerait à penser qu'elles ne sont plus vraiment incorporées. Il reste bien des chapelets et des crucifix à vendre dans la boutique de la réserve, au milieu des capteurs de rêve, des mocassins et des colliers. Des objets religieux sont donc toujours fabriqués dans les communautés, mais précisément ils n'ont ici qu'une destination commerciale de souvenirs.

Puisque les églises de mission ont participé à inscrire la mémoire semi-nomade dans la réalité sédentaire, quel rôle vont-elles être amenées à jouer maintenant que la transition est faite entre les deux modes de vie ? Qu'adviendra-t-il de l'art liturgique proprement amérindien ? Va-t-il, comme d'autres objets, être relégué au rang de souvenirs ? La mémoire du catholicisme, qui fait partie de l'histoire du semi-nomadisme, sera-t-elle perpétuée ? De par la diversité des Premières Nations autochtones du Québec, la nature des rapports mémoriels et actuels entre les Amérindiens et leur église de mission est propre à chaque communauté. L'investissement futur de ces églises devra continuer à être étudié, pour comprendre comment se réorganisent, et sont manipulés, les cadres de définition identitaires.

Marie-Pierre BOUSQUET Université de Montréal - Département d'anthropologie marie-pierre.bousquet@umontreal.ca

Anny MORISSETTE

Université de Montréal - Département d'anthropologie anny.morissette@umontreal.ca 
AnONYMe, 1969, "Bénédiction de l'église à la réserve indienne d'Amos P. Q. ", Vie Indienne, III-19, p. 8. Montréal, Commission Oblate des CEuvres Indiennes et Esquimaudes.

Appadurai Arjun, 2005, Après le colonialisme. Les conséquences culturelles de la globalisation, Paris, Payot.

Armitage Peter, 1992, «Religious Ideology Among the Innu of Eastern Quebec and Labrador ", Religiologiques, 6, pp. 63-110.

BERGERON Claude, 1987, L'architecture des églises au Québec 1940-1985, Québec, Les Presses de l'Université Laval.

Bonnemaison Joël, 1996, Les fondements géographiques d'une identité. L'archipel de Vanuatu, livre 1, Paris, Éditions de l'ORSTOM.

BOUSQUET Marie-Pierre, 2001, "Quand nous vivions dans le bois ». Le changement spatial et sa dimension générationnelle : l'exemple des Algonquins $d u$ Canada, Thèse de doctorat en cotutelle France-Québec, département d'ethnologie et de sociologie comparative, Université de Paris X-Nanterre-Département d'anthropologie, Université Laval.

-, 2005, «Les jeunes Algonquins sont-ils biculturels ? Modèles de transmission et innovations dans quelques réserves ", in Laurent JÉrôME, (éd.), Recherches amérindiennes au Québec, numéro spécial Jeunes autochtones. Espaces et expressions d'affirmation, XXXV/3, pp. 7-17.

-, 2006, "A Generation in Politics: The Alumni of the Saint-Marc-de-Figuery Residential School ", in H.C. Wolfart, (ed.), Papers of the Thirty-Seventh Algonquian Conference, Winnipeg, University of Manitoba, pp. 1-17.

Cadieux Vincent, 2003, "sans titre ", Apostolat, 74/6, pp. 10-12.

CARrière Gaston, 1958, Le roi de Betsiamites: le père Charles Arnaud o.m.i. (18261914), Ottawa, Éditions Université d'Ottawa.

-, 1963, Missionnaire sans toit: le P. Jean-Nicolas Laverlochère, o.m.i., 1811-1884, Montréal, Rayonnement, coll. "Pierres vivantes ».

-, 1978, Le père Jean-Pierre Guéguen o.m.i., Un grand voltigeur, Guérin (Québec), Éditions de la Société historique Rivière des Quinze.

CaSAult André, 2003, "House Hunting Or I've Never "Lived" in my House ", Journal de la Société pour l'étude de l'architecture au Canada/Journal of the Society for the Study of Architecture in Canada, 28/1-2, pp. 31-42.

CollignON Béatrice, 2002, "Les toponymes inuit, mémoire du territoire ", Anthropologie et Sociétés, 26/2-3, pp. 45-69.

Comaroff Jean, Comaroff John, 1991, Of Revelation and Revolution: Christianity, Colonialism, and Consciousness in South Africa, Chicago, University of Chicago Press.

Côté Rémi, 1967, "Sommes-nous des imposteurs ? ", Kerygma, I/2, pp. 69-71.

DÉSAULnIERS Laurent, 2005, "Célébrer chez les Innus », Apostolat, 76/1, pp. 16-17.

Dion Stout Madeleine, KiPLING Gregory D., 2003, Peuples autochtones, résilience et séquelles du régime des pensionnats, Ottawa, Fondation autochtone de guérison.

EDgERTON Samuel Y., 2001, Theaters of Conversion: Religious Architecture and Indian Artisans in Colonial Mexico, Albuquerque, University of New Mexico Press.

GaGnon Denis, 2002, "Les Innus de la Basse-Côte-Nord et la mission catholique de Musquaro (1800-1946) : contexte historique et tradition orale ", Recherches amérindiennes au Québec, XXXII/2, pp. 49-62. 
GÉLINAS Claude, 2002, "La création des réserves atikamekw (1895-1950) ou quand l'Indien était vraiment un Indien ", Recherches amérindiennes au Québec XXXII-2, pp. 35-48.

-, 2003a, Entre l'assommoir et le godendart. Les Atikamekw et la conquête du MoyenNord québécois 1870-1940, Montréal, Éditions du Septentrion.

-, 2003b, "Les missions catholiques chez les Atikamekw (1837-1940) : manifestations de foi et d'esprit pratique ? ", SCHEC, Études d'histoire religieuse, 69, pp. 83-99.

Gosselin Rose-Anne, 1996, Aperçu historique du vécu religieux des Algonquins au XIX ${ }^{e}$ siècle, Mémoire de maîtrise en science des religions, Montréal, UQAM.

GREEN Joyce, 2004, «Autodétermination, citoyenneté et fédéralisme : pour une relecture autochtone du palimpseste canadien ", Politique et Sociétés, 23-1, pp. 9-32.

Greer Allan, 2005, Mohawk Saint: Catherine Tekakwitha and the Jesuits, Oxford, Oxford University Press.

Grenier Denis, 15 avril 1998, «La foi chez les Amérindiens », Selon Sa Parole, 24-4, édition en ligne http://members.tripod.com/ SelonSaParole/ameri498.htm (consulté le 30 janvier 2007).

Gros-Louis Céline, Gros-Louis Charlotte, 1983, Église Notre-Dame-de-Lorette, Villagedes-Hurons, Village-des-Hurons, Musée ethnologique Arouanne.

LADNER Kiera, Orsini Michael, 2004, “De l'“infériorité négociée” à l'“inutilité de négocier” : la loi sur la gouvernance des Premières Nations et le maintien de la politique coloniale ", Politique et Sociétés, 23-1, pp. 59-87.

LALiBerté Jacques, 15 avril 1998, " Présence du renouveau charismatique dans les communautés amérindiennes ", Selon Sa Parole, 24/4, édition en ligne http://members.tripod. com/ SelonSaParole/ameri498.htm (consulté le 30 janvier 2007).

-, 2005, "Le défi est de taille », Apostolat, 76/1, pp. 6-7.

LAPOINTE Eugène, 2005, "Pour créer un leadership chrétien chez les autochtones ", Apostolat, 76-1, pp. 8-10.

Latourelle René, 1999, Jean de Brébeuf, Montréal, Bellarmin.

LAUGRAND Frédéric, 1997, “ "Ni vainqueurs, ni vaincus”. Les premières rencontres entre les chamanes inuit (angakkuit) et les missionnaires dans trois régions de l'Arctique canadien ", Anthropologie et Sociétés, 21/2-3, pp. 99-123.

Lazarus Neil, (dir.), 2006, Penser le postcolonial. Une introduction critique, Paris, Éditions Amsterdam.

Leroux Jacques, Chamberland Roland, BrazeAu Edmond, Dubé Claire, 2004, Au pays des peaux de chagrin. Occupation et exploitation territoriales à Kitcisakik (GrandLac-Victoria) au Xx ${ }^{e}$ siècle, Québec - Gatineau, Presses de l’Université Laval - Musée canadien des civilisations.

Linteau Paul-André, Durocher René, Robert Jean-Claude, 1989, Histoire du Québec contemporain, tome 1 : De la Confédération à la crise (1867-1929), Montréal, Éditions du Boréal.

Long J. Anthony, 1990, " Political Revitalization in Canadian Native Indian Societies ", Canadian Journal of Political Science/Revue canadienne de science politique, 23/4, pp. 751-773.

MARTIN Tania, 2007, «La mouvance de la culture canadienne-française vue à travers l'évolution d'une institution catholique en région ", The Canadian Historical Review, 88/1, pp. 41-88.

Mathias Joseph 'Josie', 1998, L’homme de Neawigak ... et autres histoires algonquines, Winneway, Société d'Histoire du Témiscamingue (en collaboration avec Michel Pinard). 
McNulty Gérard E., Gilbert Louis, 1981, « Attikamek (Tête de Boule) », in June Helm, (éd.), Handbook of North American Indians, 6 : Subarctic, Washington, Smithsonian Institution, pp. 208-218.

Mемm Albert, 1957, Portrait du colonisé, précédé de Portrait du colonisateur, Paris, Gallimard.

MonTPETIT Christiane, 1993, "Les Autochtones d'origine algonquine à Val d'Or: des migrants ou des citadins ", Recherches amérindiennes au Québec, XXIII/2-3, pp. 119130.

Morissette Anny, 2004, De la forêt à la réserve, la mosaïque politique d'une bande autochtone: L'exemple des Atikamekw de Manawan (Québec), Mémoire de maîtrise, Montréal, Université de Montréal-département d'anthropologie.

Noppen Luc, Morisset Lucie K., 1996, Art et architecture des églises à Québec : foi et patrie, Québec, Les Publications du Québec - ministère de la Culture et des Communications.

Nora Pierre, (dir.), 1984-1992, Les lieux de mémoire, Paris, Gallimard, [La République, 1984 ; La Nation 1, La Nation 2, La Nation 3, 1986 ; La France, 1992 : 1. Conflits et partages ; 2 . Traditions ; 3 . De l'archive à l'emblème].

Peelman Achiel, 2004, L'esprit est amérindien. Quand la religion amérindienne rencontre le christianisme, Montréal, Mediaspaul.

Pelletier Louis, 28 décembre 2006, «Paul-Émile Ottawa : personnalité de l'année 2006 », l'Action, édition en ligne http://www.laction.com/v4/article-63660-PaulEmile-Ottawapersonnalite-de-lannee-2006.html (consulté le 18 janvier 2007).

POIRIER Sylvie, 2000, "Contemporanéités autochtones, territoires et (post)colonialisme, Réflexions sur des exemples canadiens et australiens ", Anthropologie et Sociétés, 24/1, pp. 137-153.

Proulx Jean-Baptiste, 1882, Voyage au Lac Abitibi ou visite pastorale de Mgr J.-Th. Duhamel dans le Haut de l'Ottawa, Montréal, J. Chapleau et fils.

Radio-Canada, 24 septembre 2006, «Le Jour du Seigneur : Église Mission Sainte-Catherine, Pikogan ", (émission de soixante minutes).

Riopel Marc, ca 1991, Sur les traces des robes noires, Ville-Marie, Société d'histoire du Témiscamingue, coll. " Maison du Colon » 3.

SAID Edward, 1978, Orientalism. New York, Vintage.

SIMARD Jean, 2004, Le Québec pour terrain. Itinéraire d'un missionnaire du patrimoine religieux, Québec, Presses de l'Université Laval.

WiLlaime Jean-Paul, 1988, " De la sacralisation de la France. Lieux de mémoire et imaginaire national ", Archives de Sciences Sociales des Religions, 66-1, pp. 125-145.

\section{Cyberbibliographie}

"Dictionnaire biographique du Canada en ligne ", in Bibliothèque et Archives Canada, http://www.biographi.ca/FR/ShowBio.asp?BioId 34214 (consulté le 15 février 2007).

Inventaire des lieux de culte du Québec (en ligne), http://www.lieuxdeculte.qc.ca (consulté le 13 février 2007).

La Nation Atikamekw de Manawan (en ligne), http://www.manawan.org (consulté le 23 janvier 2007).

«Sur la route de Manawan », in les Collections numérisées du Canada (en ligne), http:// www.collections.ic.gc.ca/manawan.index.html (Page consultée le 23 janvier 2007). 


\section{Résumé}

Au Canada, la société québécoise, après avoir été très catholique, a connu un fort mouvement de sécularisation. Face à la désertion des lieux de culte, s'est engagé un débat sur la place des biens d'Église dans le domaine public. Qu'en est-il dans les communautés amérindiennes du Québec, qui comptent aussi chacune une église? Cet article retrace l'histoire de deux églises de missions, interrogeant leurs places dans la mémoire des oblats de Marie Immaculée et des Amérindiens algonquins et atikamekw. En étudiant les investissements symboliques des différents acteurs sociaux, les auteurs montrent que ces églises, lieux de mémoire polysémiques où l'ornementation évoque le semi-nomadisme, ont inscrit dans l'espace un modèle de type post-colonial, catholique et sédentaire. Mais, dans la réorganisation contemporaine des cadres identitaires, elles représentent aussi un début de décolonisation.

Mots-clés: Canada, Québec, Amérindiens, églises, Oblats.

\section{Abstract}

The province of Quebec in Canada is now highly secularised after a long history of Catholic domination and influence. With Churches now often deserted and unused, a discussion has emerged about the role of Church properties in the public domain. This extends to Amerindian communities in the Province, which each have their own church. This article traces the history of two mission churches and focuses on their place in the memories of the Oblate order and of the Algonquin and Atikamekw First Nations. By evaluating the symbolic investment of the major social actors, the authors show that these churches, decorated with mementoes of the semi-nomadic past, embody a post-colonial, sedentary and Catholic model. In the context of the current reorganisation and re-evaluation of identities, these churches also represent the beginnings of decolonisation.

Key words: Canada, Quebec, Native peoples, churches, Oblates.

\section{Resumen}

En Canadá la sociedad quebequense, luego de haber sido profundamente católica, fue testigo de un amplio movimiento de secularización. Ante el abandono de los espacios de culto, se ha abierto el debate en torno al papel de los bienes de la Iglesia como parte del dominio publico. Qué sucede en las comunidades indigenas de Quebec, donde cada una cuenta con una iglesia? Este artículo recupera la historia de dos iglesias misioneras, interrogándose sobre el lugar que éstas ocupan en la memoria de los Oblatos de María Inmaculada y de los indígenas algonquinos y atikamenses. Examinando las inversiones simbólicas de los diferentes actores sociales las autores muestran cómo tales iglesias, lugares polisémicos de memoria donde la ornamentación rememora el semi nomadismo, han inscripto en el espacio un modelo de tipo poscolonial, católico y sedentario. Aun así, en la reorganización contemporánea de los marcos identitarios, ellas también representan el inicio de la descolonización.

Palabras clave : Canadá, Quebec, Indígenas, iglesias, Oblatos. 